\title{
Effect of Dean Number on Heating Transfer Coefficients in an Flat Bottom Agitated Vessel
}

\author{
${ }^{1 *}$ Ashok Reddy K ${ }^{2}$ Bhagvanth Rao M ${ }^{3}$ Ram Reddy P \\ $1 * .$. Professor Dept. of Mechanical Engineering,Prof Rama Reddy College of Engineering \& Technology Nandigoan $(V)$ \\ Pantacheru(M) Greater Hyderabad A P \\ 2. Director CVSR College of Engineering Ghatkesar $(M) R R$ Dist A $P$ \\ 3 .Director Malla Reddy College of Engineering Hyderbad A P
}

\begin{abstract}
Experimental work has been reviewed using non- Newtonian and Newtonian fluids like Sodium Carboxymethal Cellulose with varying concentrations $0.05 \%, 0.1 \%, 0.15 \%$ and $0.2 \%$ with two different lengths $(\mathrm{L}=2.82 \mathrm{~m}, \mathrm{~L}=2.362 \mathrm{~m})$ and outer diameter $\mathrm{do}=6.4 \mathrm{~mm}$,inner diameter $\mathrm{di}=4.0 \mathrm{~mm}$ in an mixing vessel completely submerged helical coil. In our present study, the effect impeller speed, discharge ,flow behavior index(n) and consistency index (K) of test fluids with dimensionless curvature of the helical coil based on characteristic diameter were presented.. .
\end{abstract}

\section{Keywords: Dean Number, Curvature, Heating Rates, Agitated Vessel}

\section{Introduction}

To improve the performance of heat transfer rates, the fundamental aspect of flow patterns is an essential and important criteria in the helical coil design theory. The helical coil silent features consists of a inner diameter di and mean coil diameter Dm which is also known as pitch circle diameter of the coil. The ratio of coil pipe diameter to the mean coil diameter is known as dimensionless curvature .The ratio of pitch of the helical coil to the developed length of one turn is called non dimensional pitch .The angle which makes with plane geometry perpendicular to the axis of the one turn of the coil dimensions ic defined as helix angle.

Two fundamental theories put forwarded by Dean[1] and Mortan[2] describes the flow patterns in the inner side of the tube. In the coordinate geometry of the coil diameter, the secondary velocity field is known to be Dean Vortex. Two Cyclonic flow field occurs and one which is generated away from the axial centre line is quantify by the dean number which in turn defines the viscous forces dominating along the surface of the coil. The dean

number described by Dravid et al [3] as De=Re $(\mathrm{do} / \mathrm{Dm})^{0.5}$ where do is equal to outer diameter of the coil tube and $\mathrm{Dm}=\mathrm{mean}$ diameter of the coil and Re Reynolds number. Morton Vortex is generated due the buoyancy effects which in turn consists of two vertical separated vertical cyclonic flow field .

Seban and Mclaughin [4] suggested that the critical Reynolds number for helical coil is found to be higher compared that of straight tube .The authors also pointed out the centrifugal forces are higher in case of helical coil than the straight tube. Lee et al [5] study the buoyancy effects with heat flux to quantify the Grashoff number and later tainted that the gravitational forces specify that when central fluid particles comes down while the less dense fluid particles moves up against the surface wall of the coil. The author verified or made alterations in secondary flow by educing the curvature effect with constant Dean number and Grashoff number.Lin and Ebadian [6] study the effect of fully developed turbulent k- $\varepsilon$ model at the entrance of the coil using finite volume method with CFD Code including the Dean number and Grashoff number.

Huttl and Friedrich [7] noticed turbulent nature in helical coil using the Navier-Stokes equations and assuming the orthogonal coordinate geometry plane. The secondary flow field distribution in the inlet region of the coil pipe spreads over the coil pipe ant the average pressure gradient shows linear giving the maximum and minimum at the outer and inner wall tube surface. There were no pressure gradient exits at the centre of moving along the surface in the secondary flow field which inviolate the earlier conclusion to vortices formation in the velocity field. .

One can refer the previous papers [8] for details of the experimental setup and procedure for conducting the experimental runs in our present works. The experiments were carried with curvature ratio d/D with 0.02564 ; where $\mathrm{d}$ is equal to inner diameter of the coil and $\mathrm{D}=$ constant mean helical coil diameter . 
IOSR Journal of Engineering

May. 2012, Vol. 2(5) pp: 945-951

\section{Mathematical Formulations}

Inner and outer diameter of the coil pipe for experimental overall heat transfer coefficient are determined using the relation as [8]:

$Q^{*} \rho^{*} \mathbf{C}_{\mathbf{p}} *\left(\mathbf{T}_{\mathbf{0}}-\mathbf{T}_{\mathrm{i}}\right)=\mathbf{U} \cdot \mathbf{A}^{*}\left(\mathbf{T}_{\mathrm{b}}-\left(\mathbf{T}_{\mathbf{0}+} \mathbf{T}_{\mathrm{i}} / 2\right)\right.$

The Properties density $\rho$ and specific heat $C_{p}$ are calculated for above equations at the mean temperature of inlet $\left(T_{i}\right)$ and maximum outlet temperature of the coil test solution $\mathrm{T}_{\text {omax. }}$ for any heating data experiment.

Time average overall heat transfer coefficient for heating data was calculated by the following equation[8]:

$$
\overline{\mathbf{U}}_{\text {avg. }}=\sum \mathbf{U}_{\mathbf{p}} \mathbf{t}_{\mathrm{p}} \Delta \mathbf{t}_{\mathrm{p}} / \sum \mathbf{t}_{\mathbf{p}} \Delta \mathbf{t}_{\mathrm{p}}
$$

The equations were developed using regression analysis from shear stress-shear rate experimental results obtained for four $0.05 \%, 0.1 \%, 0.15 \%$ and $0.2 \%$ Sodium Carboxymethal Cellulose in water at different temperatures used in our present study Newtonian fluids with $0.05 \% \mathrm{CMC}$ and $0.1 \% \mathrm{CMC}$, the equation is written as

$\mathrm{Nu}=0.0622\left(\right.$ Dei) ${ }^{0.5}(\operatorname{Pr})^{0.1}$

Range :Dei=7000-55000 Pr=0.15-1.5

Non-Newtonian fluids with $0.15 \% \mathrm{CMC}$ and $0.2 \% \mathrm{CMC}$, the equation is given by

$\mathrm{Nu}=0.255(\text { Dei })^{0.5}(\operatorname{Pr})^{0.1}$

Range: Dei=300-2500 Pr=0.8-4.5

Using equations(3)-(4), the Nusselt number have been estimated The Dean number is equal to $\operatorname{Re}(\mathrm{d} / \mathrm{D})^{0.5}$ where $\mathrm{d} / \mathrm{D}$ is 0.02564 . The equation of Reynolds number $\mathrm{Re}=\mathrm{D}_{\mathrm{i}}^{2} \mathrm{~N} \rho / \mu_{\text {avg. }} \& \mathrm{Re}=\mathrm{dV} \rho / \mu_{\text {avg }}$ were used to evaluate $0.05 \%$ and $0.1 \% \quad \mathrm{CMC}$ test solutions for outer and inner diameter of the helical coil To show the effect of flow behavior index and consistency index on heating rates of $0.15 \%$ and $0.2 \% \mathrm{CMC}$ test solutions, a modified Reynolds number is $\operatorname{Reo}=(\mathrm{NDi})^{2-\mathrm{n}} \mathrm{Di}^{\mathrm{n}} \rho / \mathrm{K}$ and $\operatorname{Re}=\mathrm{Di}^{\mathrm{n}} \mathrm{V}^{2-\mathrm{n}}$ $\rho / K / 8[6 n+1 / n]^{n}$ were used.

The Prandtl Number was estimated as $\operatorname{Pr}=\mathrm{Cp} \mu / \mathrm{k}$ for four different $\mathrm{CMC}$ test Solutions for outer diameter of the coil. For inner diameter of the coil the relation used to determined is equal to $\operatorname{Pr}=\mathrm{K} \mathrm{Cp}(\mathrm{V} / \mathrm{di})^{\mathrm{n}-1} / \mathrm{k}$ This relation was used to know the behavior of consistency index on heat transfer coefficients. The coefficients a and $b$ in the above equation(9) were taken from the published work contributed by Kelb and Seader[9] theoretical model for curved tube. The coefficients given by them are $a=0.5$ and $b=0.1$. These coefficients were used to evaluate the Dean nimber and Prandtl number for four different CMC test solutions. The equation proposed by them is written as $\quad \mathrm{Nu}=0.836 \mathrm{De}^{0.5} \operatorname{Pr}^{0.1} \mathrm{De}>80 \quad 0.7>\operatorname{Pr}>5$. The range of Dean number and Prandtl number obtained from experimental results for heating data in our present study is good agreement with the range obtained by Kelb and Seader[9].

\section{Results and Discussion}

Figure 1 shows the effect of impeller speed on the Dean number with curvature d/D equal to 0.02564 for $0.15 \%$ and $0.2 \% \mathrm{CMC}$ test solutions. As speed of the impeller increases, the dean number values also shown increasing trend. Similar trend has been noticed for $0.05 \% \mathrm{CMC}$ and $0.1 \% \mathrm{CMC}$ test solutions used in our experimental runs.

It is evident that the increase in impeller speed $\mathrm{N}=300 \mathrm{rpm}$, the Dean number also increases for $0.2 \% \mathrm{CMC}$ test solution, at impeller speed $\mathrm{N}=65 \mathrm{pm}$, for $0.15 \% \mathrm{CMC}$, the Dean number increases as the impeller speed increase further upto $\mathrm{N}=200 \mathrm{rpm}$. The effect of impeller speed on Dean number can be visualized from the Figure-1. 
IOSR Journal of Engineering

May. 2012, Vol. 2(5) pp: 945-951

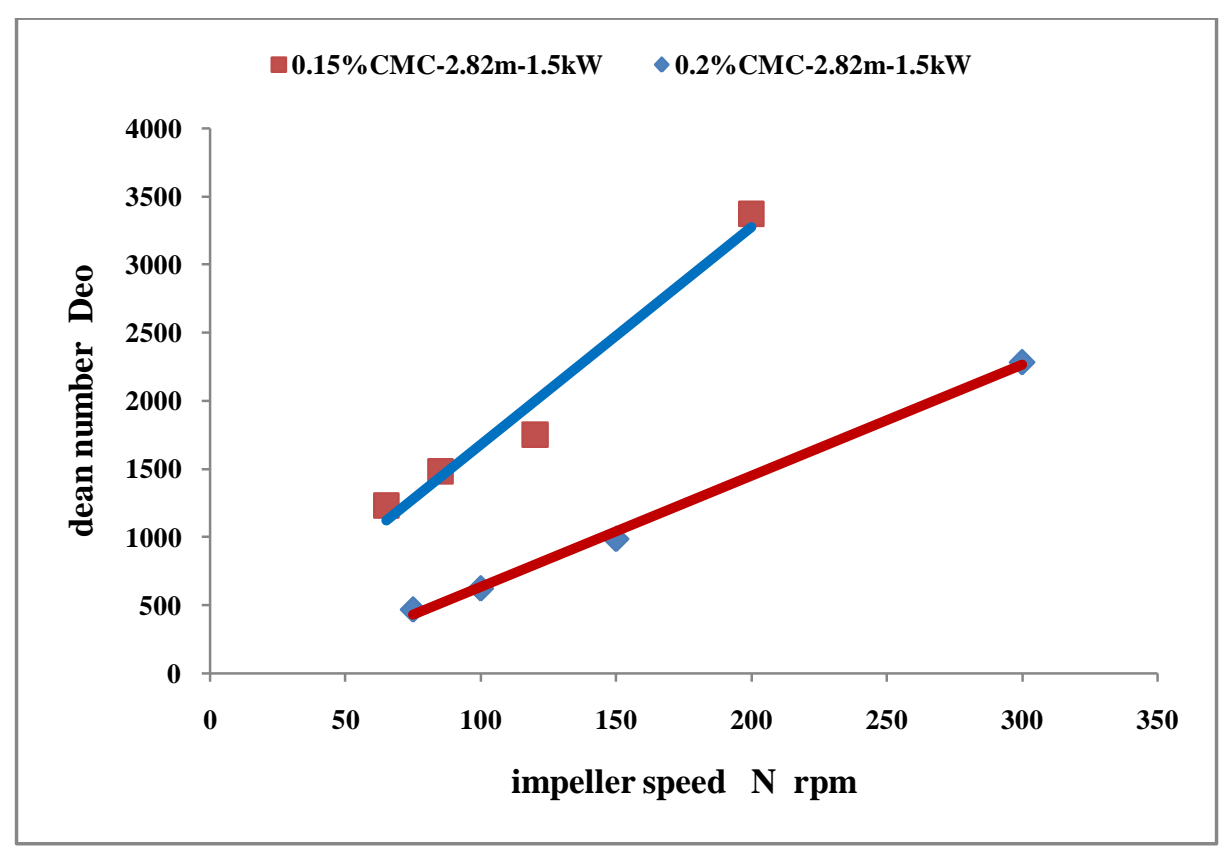

\section{Figure-1 Effect of Impeller Speed on Dean number of non-Newtonian Fluids N=300rpm for 0.2\% CMC , N=200rpm fpr 0.15\% CMC.}

At impeller speed N=60rpm, increased in Dean number was noticed for 0.05\%CMC and 0.1\%CMC test solutions. One can make conclusion from the above fact that the increase in Dean number depends not only the impeller speed but also it depends on other variables like flow rate, viscosity of the fluid and the \% of concentration of the test solutions used in experimental runs.

.The effect of flow behavior index on dean number indicates as flow behavior index is increased, the dean number get decreasing. The flow behavior index depends on temperature and the fact may be may be due to the controlling the flow rate and the mean temperatures of the test solutions flowing through the helical coil inlet. The flow behavior index has been calculated for average temperature of inlet and maximum outlet temperature of the coil $(\mathrm{L}=2.82 \mathrm{~m}, 1.5 \mathrm{~kW}$ input and $0.2 \% \mathrm{CMC}$ and shown in the Figure-2.

In Figure-3 we could observe the influence of the consistency index K on the Dean number. The Dean number increase with increasing consistency index. This fact may be due the decreasing mean temperatures were used to evaluated the Dean number for four different concentrations in our experimental studies.

In an mixing vessel, the rheological properties of the test fluids to be used are of main important regarding the parameters which have to be considered for the configuration. The viscosity is the most important characteristic value. While Newtonian fluids are characterized by a constant viscosity, on other hand the non-Newtonian fluids demands the consideration of a changing viscosity through a viscosity function. 
IOSR Journal of Engineering

May. 2012, Vol. 2(5) pp: 945-951

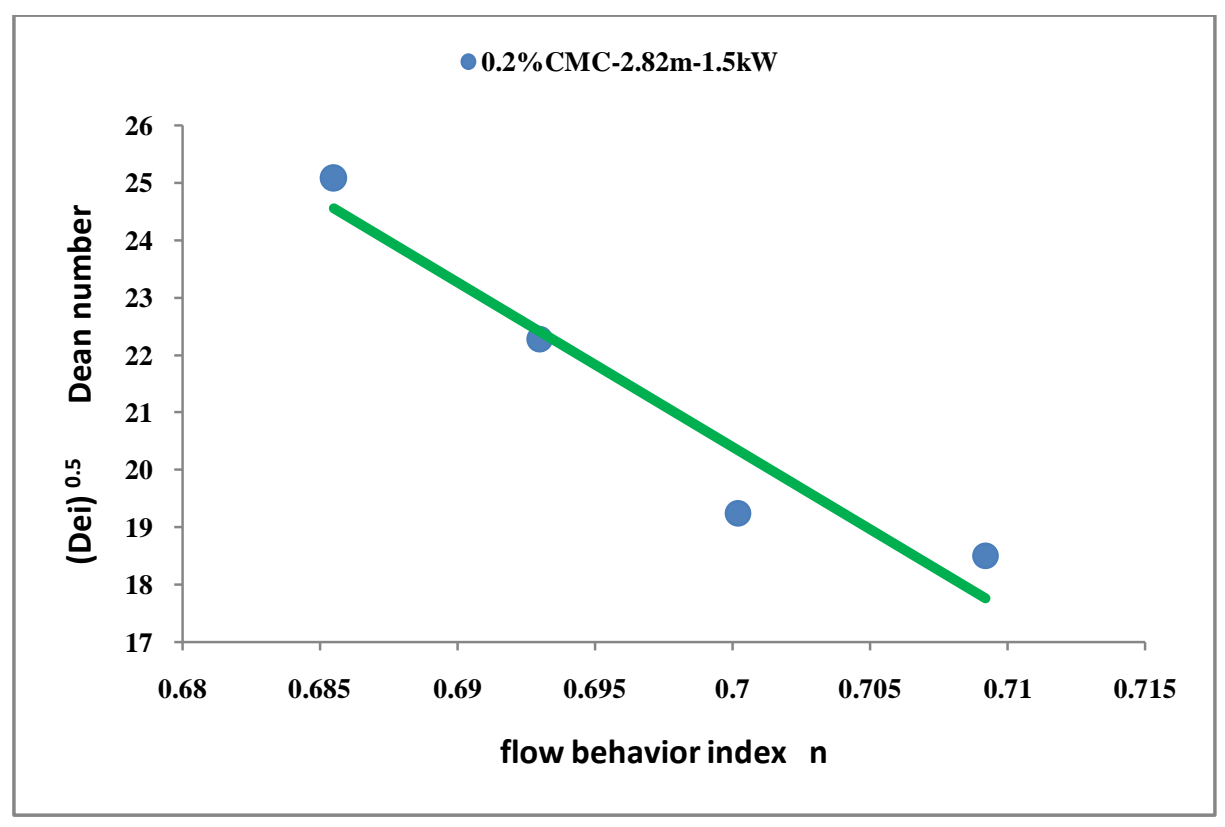

Figure-2 Effect of flow behavior index on Dean number

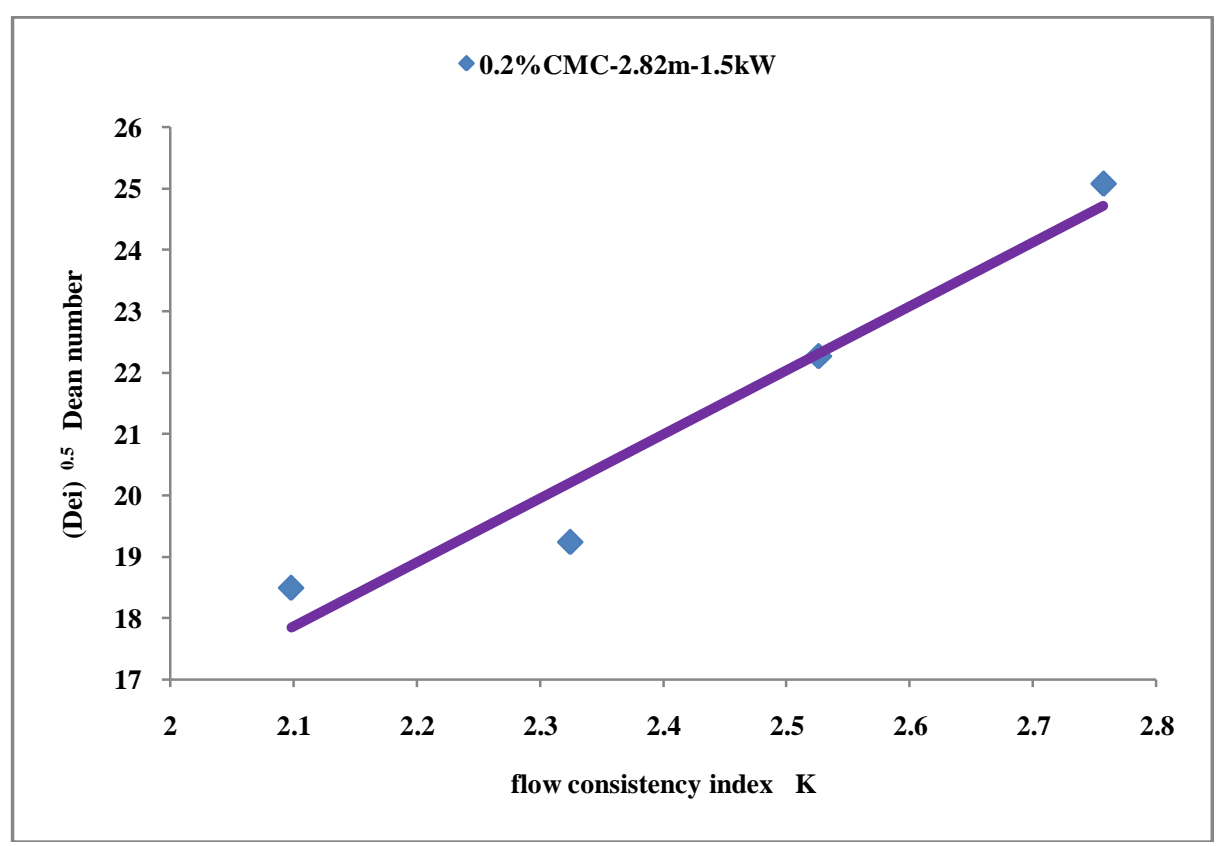

Figure-3 Effect of flow consistency index on Dean number

The consistency index $\mathrm{K}$ is a strong function of viscosity and hence the non-Newtonian test fluids have been evaluated using consistency index instead of viscosity relations.

Figure-4 indicates the effect of curvature ratio that is d/D which is incorporated in the dimensionless Reynolds number and to evaluate the dean number. The correction factor used by Dean[1] in the flow field in the Reynolds number is to overcome the centrifugal forces existing in the secondary flow along the surface of the coil. We have assumed the predicated values of the curvature ratio that is as $, \boldsymbol{\delta}_{\mathbf{1}}=\mathbf{0 . 0 3 8 4 6}, \boldsymbol{\delta}_{2}=\mathbf{0 . 0 5 1 2 8 2}, \boldsymbol{\delta}_{3}=\mathbf{0 . 0 6 4 1 0 2}$ and $\boldsymbol{\delta}_{\mathbf{4}}=\mathbf{0 . 0 7 6 9 2 3}$. These ratios were utilized for evaluating the predicated dean number for two different lengths of the coils that is $\mathrm{L}=2.362 \mathrm{~m}$ and $\mathrm{L}=2.82 \mathrm{~m}$ and four different concentrations. It can be visualized from the Figure-4 that the correlated Nusselt number increases with increase in curvature ratio dean number evaluated at different flow rates of non-Newtonian fluids. Similar trend is observed for Newtonian fluids 
IOSR Journal of Engineering

May. 2012, Vol. 2(5) pp: 945-951

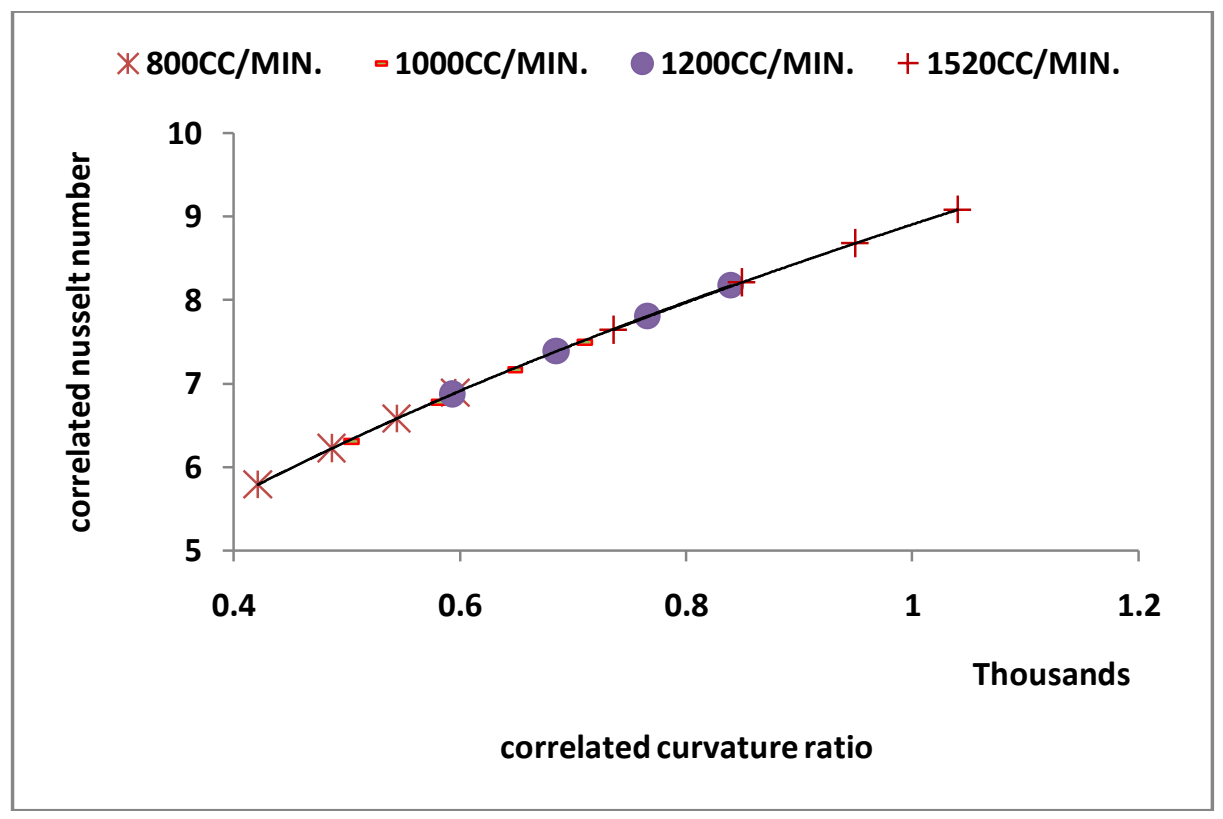

Figure-4 Effect of Curvature Ratio on Correlated Nusselt Number for 0.2\% CMC at Different Flow Rtaes L=2.362m Heat Input=1.5kW-Non-Newtonian Fluids

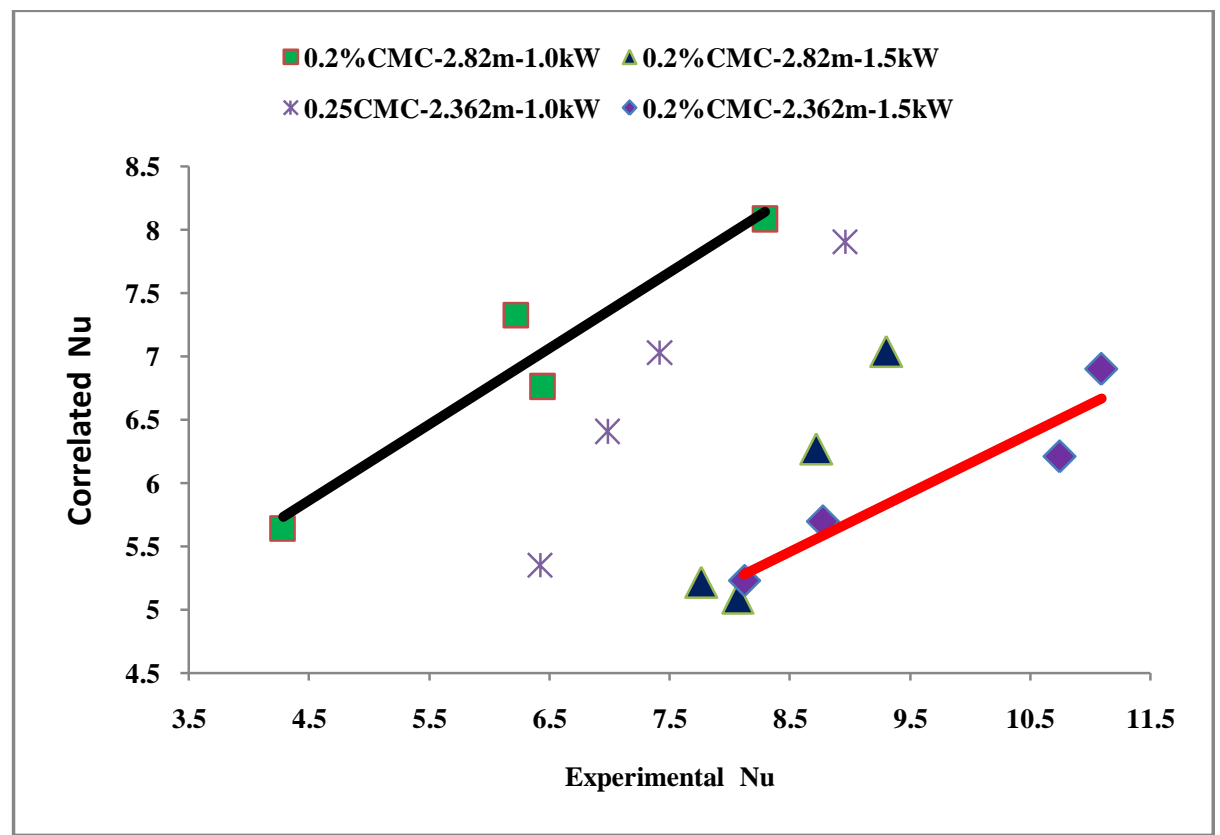

Figure-5 Comparison of Experimental and Correlated Nusselt number for non- Newtonian Fluids

It is seen from the experimental observations that as the heating of the constant test fluid in the mixing vessel is increased from $1.0 \mathrm{~kW}$ to $1.5 \mathrm{~kW}$ using kanthal heating elements ,the bulk temperature of the test fluid got increased. Due to increase in temperature difference, the Dean number also gets increased. The Dean number evaluated for inner diameter of the coil showed no noticeable variation compared with Dean number calculated for outer diameter of the coil. Hence, in our present study, we have presented results obtained from dimensionless Dean number, Prandtl number and Nusselt number for inner diameter of the coil

The experimental results obtained have been evaluated for Dean number and Prandlt number for different lengths of the coil $\mathrm{L}=2.82 \mathrm{~m}$ and $\mathrm{L}=2.362 \mathrm{~m}$ with Heat Inputs of $1.0 \mathrm{~kW}$ and $1.5 \mathrm{~kW}$ and inner coil pipe diameter $=4.0 \mathrm{~mm}$ with curvature ratio $\mathrm{d} / \mathrm{D}$ 
IOSR Journal of Engineering

May. 2012, Vol. 2(5) pp: 945-951

$=0.02564$ for both Newtonian and non-Newtonian Fluids and tabulated in Table-1 \& Table- 2 The $\%$ of error of experimental results obtained for Nusselt number compared to theoretical results are found to be on an average of $15.9 \%$. as shown in Figure-

\section{Conclusions:}

1.The influence of impeller speed, flow behavior index, consistency index and curvature ratio on Nusselt number was studied using paddle impeller and low shear rates test solutions in an agitated vessel

2..The viscosity data obtained was used to classify the Newtonian and non-Newtonian test solutions and best fit power law models were developed

3..By fixing the exponent values of the Dean number and Prandtl number in equations (3) \&(4), we were able to get one parameter fit of the remaining constant c for both Newtonian and non-Newtonian fluids.

\section{Acknowledgements:}

The authors would like to express their appreciation to the Prof. Rama Reddy College of Engineering \& Technology Hyderabad A P for providing the facilities to carry out this research work.

Table-1 0.05\% CMC, 0.1\% CMC L $=2.82 \mathrm{~m}, \mathrm{~L}=2.362 \mathrm{~m} 1.0 \mathrm{~kW}, 1.5 \mathrm{~kW}$ Dean Number Prandlt Number \& Nusselt Number-Newtonian Fluids

\begin{tabular}{|c|c|c|c|c|c|c|c|}
\hline $2.82 \mathrm{~m}$ & $1.0 \mathrm{~kW}$ & $0.05 \%$ & & & $0.1 \%$ & & \\
\hline F.R & Dei & Pr & $\mathrm{Nu}$ & F.R & Dei & Pr & $\mathrm{Nu}$ \\
\hline 960 & 30104.258 & 0.1677 & 7.9935 & 800 & 7330.081 & 0.5721 & 5.6134 \\
\hline 1080 & 33699.284 & 0.1689 & 7.9987 & 1200 & 10631.909 & $\mathbf{0 . 5 9 3 3}$ & 8.333 \\
\hline 1440 & 44612.953 & 0.1705 & 8.1867 & 1600 & 13046.919 & 0.6483 & 7.8606 \\
\hline 1640 & 50445.633 & 0.1721 & 8.8211 & 1950 & 14734.008 & $\mathbf{0 . 7 0 3 3}$ & 7.7062 \\
\hline $2.82 \mathrm{~m}$ & $1.5 \mathrm{~kW}$ & $0.05 \%$ & \multicolumn{5}{|c|}{$0.1 \%$} \\
\hline 1240 & 37904.091 & 0.1736 & 7.8121 & 1000 & 8306.374 & 0.6378 & 7.0205 \\
\hline 1440 & 41694.458 & 0.1863 & 7.64 & 1200 & 8799.459 & 0.7346 & 8.2469 \\
\hline 1520 & 43288.506 & 0.1903 & 8.1842 & 1760 & 12120.167 & 0.7868 & 8.8933 \\
\hline 1840 & 52149.902 & 0.1915 & 9.026 & 1920 & $\mathbf{1 3 0 4 0 . 8 3}$ & 0.7989 & 9.3294 \\
\hline $2.362 \mathrm{~m}$ & $1.0 \mathrm{~kW}$ & $0.05 \%$ & \multicolumn{5}{|c|}{$0.1 \%$} \\
\hline 960 & 30461.832 & 0.165 & 7.497 & 800 & 7078.348 & 0.5917 & 5.2766 \\
\hline 1080 & 33665.337 & 0.1691 & 9.8973 & 1200 & 9190.745 & 0.6914 & 6.7571 \\
\hline 1440 & 43411.51 & 0.1768 & 8.2087 & 1600 & 11516.007 & 0.7433 & 9.1253 \\
\hline 1640 & $\mathbf{4 8 7 9 7 . 9 7}$ & 0.1798 & 9.6618 & 1950 & 13832.565 & 0.7567 & 7.594 \\
\hline $2.362 \mathrm{~m}$ & $1.5 \mathrm{~kW}$ & $0.05 \%$ & \multicolumn{5}{|c|}{$0.1 \%$} \\
\hline 1240 & 38062.931 & 0.1726 & 8.6292 & 1000 & 8364.007 & 0.6326 & 5.8072 \\
\hline 1440 & 42369.514 & 0.1825 & 8.787 & 1200 & 8997.025 & 0.7156 & 6.0612 \\
\hline 1520 & 43702.446 & 0.188 & 9.5497 & 1760 & 12669.093 & 0.7499 & 8.5809 \\
\hline 1840 & 51196.514 & 0.196 & 10.0471 & 1950 & 13385.882 & 0.7797 & 7.672 \\
\hline
\end{tabular}


Table-2 0.15\% CMC, 0.2\%CMC L=2.82m,L=2.362m 1.0kW,1.5kW Dean Number Prandlt Number \& Nusselt Number-Non-Newtonian Fluids

\begin{tabular}{|c|c|c|c|c|c|c|c|}
\hline $2.82 \mathrm{~m}$ & $1.0 \mathrm{~kW}$ & $0.15 \%$ & & & $0.2 \%$ & & \\
\hline F.R & Dei & Pr & $\mathrm{Nu}$ & F.R & Dei & Pr & $\mathrm{Nu}$ \\
\hline 1080 & 1365.6201 & 1.131 & 7.5185 & 920 & 401.9557 & 2.6694 & 4.2836 \\
\hline 1360 & 1609.4352 & 1.1504 & 7.2671 & 1320 & $\mathbf{5 8 2 . 5 5 5 2}$ & 2.5541 & 6.4436 \\
\hline 1440 & 1720.2729 & 1.137 & 9.312 & 1560 & 686.3622 & 2.5102 & 6.222 \\
\hline 1720 & 1844.2181 & 1.1581 & 8.3853 & 1950 & 837.8086 & 2.4831 & 8.2925 \\
\hline $2.82 \mathrm{~m}$ & $1.5 \mathrm{~kW}$ & $0.15 \%$ & & & $0.2 \%$ & & \\
\hline 1000 & 1398.3726 & $\mathbf{1 . 0 7 1 5}$ & 6.7765 & 800 & 342.0254 & 2.7451 & 7.763 \\
\hline 1400 & 1850.6423 & 1.0729 & 6.9488 & 1000 & 326.524 & 2.7194 & 8.0651 \\
\hline 1680 & 1808.3748 & 1.1908 & 7.5147 & 1200 & 495.8837 & 2.6914 & 8.7191 \\
\hline 1880 & 2083.0748 & 1.1557 & 8.0978 & 1520 & 628.9264 & 2.6188 & 9.3015 \\
\hline $2.362 \mathrm{~m}$ & $1.0 k W$ & $0.15 \%$ & & & $0.2 \%$ & & \\
\hline 1080 & 1315.9388 & 1.1577 & 7.6262 & 920 & 356.7285 & 2.8497 & 6.4218 \\
\hline 1360 & 1688.6346 & 1.1176 & 8.0701 & 1320 & 514.7285 & 2.7658 & 6.985 \\
\hline 1440 & 1762.1533 & 1.1206 & 8.0586 & 1560 & 624.2632 & 2.6671 & 7.418 \\
\hline 1720 & 2187.9425 & $\overline{1.0742}$ & 8.3646 & 1950 & 795.8171 & 2.5668 & 8.9633 \\
\hline $2.362 \mathrm{~m}$ & $1.5 \mathrm{~kW}$ & $0.15 \%$ & & & $0.2 \%$ & & \\
\hline 1000 & 1207.6354 & 1.1728 & 6.8401 & 800 & 344.0077 & 2.7348 & 8.12 \\
\hline 1400 & 1352.4596 & 1.2195 & 7.2236 & 1000 & 406.5245 & 2.7745 & 8.7764 \\
\hline 1680 & 1873.2316 & 1.1658 & 8.3212 & 1200 & 484.5153 & 2.7319 & 10.7439 \\
\hline 1880 & 1712.8551 & 1.18 & 8.5715 & 1520 & 600.4778 & 2.6981 & 10.0933 \\
\hline
\end{tabular}

\section{References:}

1) W R Dean The Stream Motion in a Curved Pipe Philios Magazine 201928 p208

2) B R Morton Turbulences Flow Field Studies J Fluid Mechanics 51959 p151

3) AN Dravid KA Simth EW Merrill PLT Brain Effect of Secondary Fluid on Laminar Flow Heat Transfer in Helically Coiled Tubes AIChE J 171971 p1114.

4) RA Seban \& EF Mclaughlin Heat Transfer in Tube Coils with Laminar and Turbulent Flow International Journal Heat Mass Transfer 61963 p387

5) JB Lee HA Simon \& JCF Chow Buoyancy in Developed Laminar Curved Tube Flows International Journal Heat Mass Transfer 281985 p631

6) CX Lin MA Ebadian The Effects of Inlet Turbulence on Development of Flow and Heat Transfer in Helically Coiled Pipe. Int. J. Heat Mass Transfer 421999 p739

7) TJ Huttl R Friedrich Influence of Curvature and Torsion on Turbulent Flow in Helically Coiled Pipes. International Journal Heat Fluid Flow 212000 p345

8) K Ashok Reddy Heat Transfer to Non-Newtonian Fluids in an Agitated Vessel Using Paddle Impeller PhD Thesis J N T University Hyderabad

9) CE Kalb JD Seader Fully Developed Viscous Flow Heat Transfer in Curved Circular Tubes with Uniform Wall Temperature AIchE Journal 201974 p340 